\title{
Responses to the Letter: Cervical Foraminal and Discal Height after Dynamic Rotational Plating in the Cervical Discectomy and Fusion
}

\author{
Jin-Oh Park ${ }^{1}$, Moon Soo Park ${ }^{2}$, Seong-Hwan Moon ${ }^{3}$, In-Sung Kim², Seok Woo Kim², \\ Yong-Chan Kim², Tae-Hwan Kim², Bo-Kyung Suh ${ }^{2}$, Ji Hoon Nam², Hwan-Mo Lee ${ }^{3}$ \\ ${ }^{1}$ Department of Orthopaedic Surgery, Yongin Severance Hospital, Yonsei University College of Medicine, Yongin, Korea \\ ${ }^{2}$ Department of Orthopaedic Surgery, Hallym University Sacred Heart Hospital, Hallym University College of Medicine, Anyang, Korea \\ ${ }^{3}$ Department of Orthopaedic Surgery, Yonsei University College of Medicine, Seoul, Korea
}

I agree with you. You rightly pointed out the potential weakness of rotational dynamic plates. From your comment, most studies have been on translational dynamic plates, and unfortunately only a few studies have concerned the use of rotational dynamic plates. My guess is that the rotational dynamic plates may have less dynamic motion than the translational ones and with more dynamic motion than the static translational plates due to the screw-bone interfaces of the rotational dynamic plates adding instability.

\section{Conflict of Interest}

No potential conflict of interest relevant to this article was reported. 